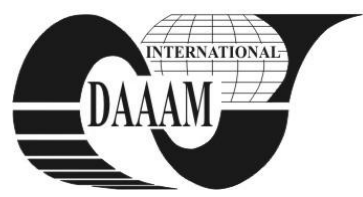

Annals of DAAAM for 2011 \& Proceedings of the 22nd International DAAAM Symposium, Volume 22, No. 1, ISSN 1726-9679 ISBN 978-3-901509-83-4, Editor B. Katalinic, Published by DAAAM International, Vienna, Austria, EU, 2011 Make Harmony between Technology and Nature, and Your Mind will Fly Free as a Bird Annals \& Proceedings of DAAAM International 2011

\title{
MOBILE APPLICATIONS TESTING
}

\section{SEKANINA, A[les]; PROKOPOVA, Z[denka] \& SILHAVY, R[adek]}

\begin{abstract}
The purpose of this paper is to introduce the importancy of the testing in a mobile application developing process. The applications without testing can be dysfunctional, unusable and unsecured. The possibility of data loss can have disastrous consequences for a particular application segment. In this paper the testing methods and benefits of the testing are described. The final part of the paper describes how to develop applications with testing effectively.
\end{abstract}

Key words: mobile application, testing, usability

\section{INTRODUCTION}

A mobile phones and tablets predominate on the field of personal computers. The application development is is focused to these devices more than to typical software development for computers. Applications are less complex as software for computers, so their development takes less time. However, in accelerating of these products are not fully functional and usable. Applications without feedback, thus testing rarely succeeds on the market.

The Software testing is still underestimated part of an application and software development. The introduction of software testing and quality control can reduce the cost and time frame for deliverying of the projects. Computer users are sometimes obliged to use software with bugs, because the have no choice to similar application. There may not be mistakes in mobile applications. The Applications must be stable, secure, performance and usable.

\section{ISSUES TO MOBILE APPLICATIONS}

The developers of mobile applications must solve several issues in the development of applications. Mobile device manufactures, continually produced new devices with new properities. It is necessary to adapt applications to those devices. Differences may be, for example in the resolution, the position of navigation and navigation buttons. Devices are changing frequently and the application must constantly be tested on new platforms.

There are various operating systems used in mobile devices. Therefore creation one application that will run on all devices is not possible. It has to be developed unique version of the application for each version of the operating system. This requires knowledge of the programming codes and diffrerent approach to the software testing, especially white-box testing. This testing method is described below.

Mobile devices already have the same or very similar properties, such as computial power, Internet availability and others. Mobile into two types: a full touch devices and devices with hardware keyboard. This has an impact on the creation of the user interface. If applications are developed for Symbian, a developer have to respect that there full touch devices and devices with the separate keyboard.

The last one is type of application. Applications are divided into native and web applications. The native applications are programmed as an application for each platform. Web application is available for all devices. It runs on the server, to which devices are connected to.

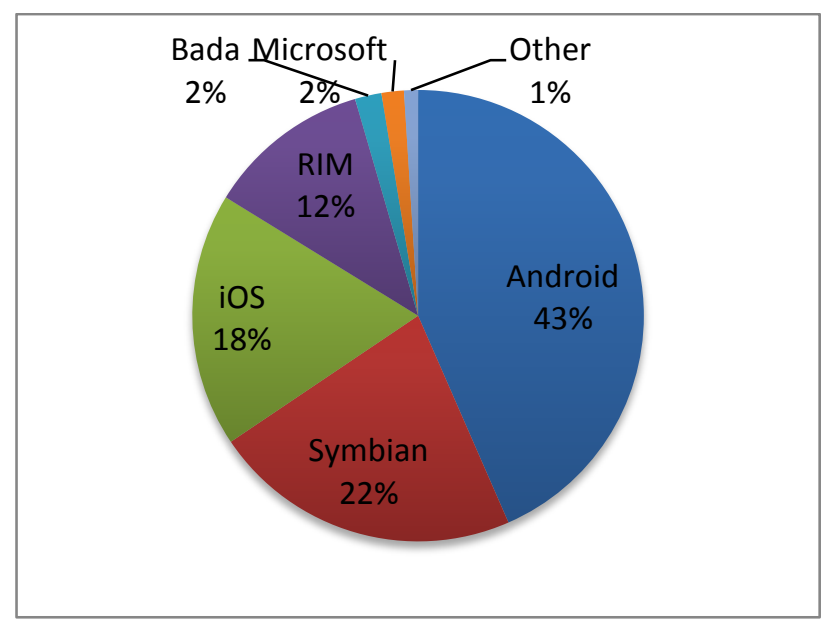

Fig. 1. Smartphone OS market share in Q2 2011(Gartner, 2011)

\section{UNIT TESTING}

Unit testing involves testing the smallest possible unit of a programme code. (Budrovich, 2001) These smallest possible units can be for example classes or data structures. Testing functional units differ from the unit testing. If the unit testing is omitted, there might begin large time lags to produce correct mobile application.

\section{MOBILE APPLICATION USABILITY}

The goal of an usability test is to identify actionable faults in the system from subjective user perspective (Fling, 2009) Usability is the one of the most important features for users. They do not inspect the application source code. They only use interface of the application. Automated tests do not show if the usability is appropriate or not. Only users may evaluate this fact. Usability can be tested throughhoug throughout nebo during the whole development process. The most appropriate approach is to test early and often. It is need to select targeted group especially potentional customers for usability testing. Usability may be different due to different platforms.

\subsection{Usability testing workflow}

- Page layout - testers examine the overall appereance and effectiveness of the application. They discover whether they are instinctively directed to the main functions of the application and whether key featuers are not missing.

- Color schemes - Use of colors on background, texts, icons, buttons and other elements in application.

- Findability - Testing scenario should also includes tasks where users have to find some useful functions. It may be 
for example Help, Application instructions, Search engine and others. This testing is very helpful to determine accessibility.

- Language localization - There have to be tested texts, messages and symbols in an application translated into multiple languages.

These points describe the main part of an application.

However it is need to test all details eg. navigations, headers, fonts, error messages, use of keypad and other. Good usability is an advantage against competition applications.

\section{FUNCTIONAL TESTING}

Functional testing, referred also as black-box testing is possible to use when modules are treated as functional elements for users. Tester gives inputs to module and gets outputs. Functional testing examines wether the output is correct. This testing is performed on devices with real conditions, ie. smart phones. To perform black-box testing, it is need a test plan made up of a road map for testing along with specific test cases that specify what is to be tested. Persons can do functional testing without programming skills. It is possible to test huge systems in a short time. Tester can make test scenarios. Tester clearly defines what will be tested. Testing does not depend on the actual conditions. Although the conditions for testing, like other network, will change, is tested as well. It is not neccesarry to change test scenarios.

\section{WHITE-BOX TESTING}

The purpose of white-box testing is to find hidden issues that may not be exposed by black-box testing. The goal of white-box testing is to find violations of coding standards, and to expose other problems that only reveal themselves by looking at the execution of the code. The white-box testing can prove to be invaluable in the user interface development. This is the case if a layered approach such as building a generic user interface is used. In this case, if there is a bug in the generic user interface, it is more easily discovered with a white-box test.

\section{TESTING PROCESS}

As it was written above, the testing is composed of several tests. The developers usually apply testing at the end of the process. Standart process is shown on the Fig. 2. Developers must return to the beginning and go throw the whole process again if the results are bad. This significantly increases time of the application development. The result is often the application with bugs that the developers fix in other versions. However there are a lot of opportunities of applications on the market and user already may not have so much patience. In addition to increasing delay arise financial costs of the project. Repeated designing, programming and testing can increase development costs many times.

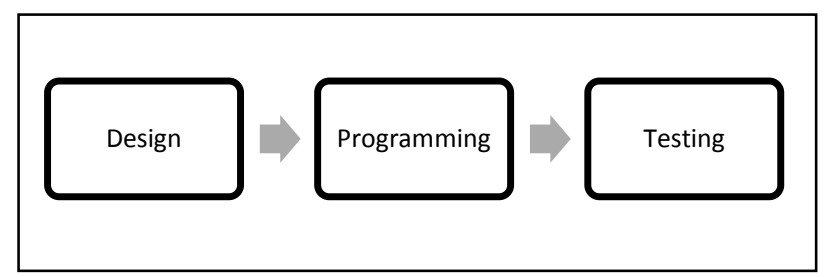

Fig. 2. Standart development process

It is possible to user mobile application development tools for faster and better development. These development tools already include programming frameworks throw crossplatforms. The framework is software structure that serves as support for programming and development organization. Framework can include programme codes, a library application programming interface, design patterns or best practices for development. These features can decrease time of the developing and testing. Debugging tools is also implemented in development tools. Therefore it is not necessary to test a code in the real mobile. Testing should take place throughout the developing proccess, as is show on Fig.3.

Design and usability of application can be tested from the draft on the paper. Testing the code begins with unit testing and further testing of the overall code. It has been going on in mobile application development tools where is debugging tools. It is possible to use emulators. The emulators simulate specific mobile devices directly on the computer. There is no need to record application in device for each testing. The emulators are able to test both graphical interface and application functionality.

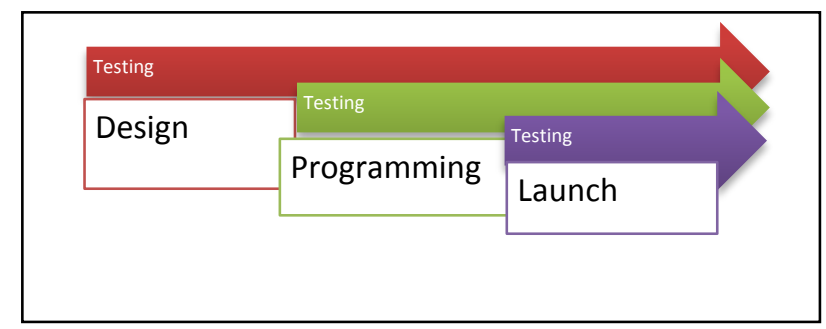

Fig.3. Better development process

\section{CONLUSION}

Mobile devices predominate on the computer market. Hundreds of the applications are increasing every day in app stores. The developers are now more focused on these devices. The mobile applications are developed, both offline and online. Webdevelopers also focuses its development on the mobile version of websites. All applications must be simple and easy to use which is not easy to do. The testing of products is much more likely that these applications will be functional and simple.

Since the testing takes almost the same time as application development, it is apropiete to use development tools that enable faster, clearer and easier development. These development tools including programming frameworks that facilitate programming and debugging tools with both test quality and functionality of the application code.

It is suitable to use emulators for functional testing. The emulators simulate specific mobile devices on the computer. Testing can take place through the process of development and this may result in quality software.

\section{REFERENCES}

Fling, B. (2009). Mobile design and development, O'reilly Media, Inc., ISBN 978-0-596-15544-5, Sebastopol

B'Far, R. (2005). Mobile computing principles, Cambridge University Press, ISBN 978-0-511-26576-1, New York

Budrovich, V. (2001). Techniques for Preventing Wireless System Software Errors, Proceedings of Wireless One, SIGS 101 Conference

Gartner. (2011). http://gartner.com/it/page.jsp?id=1764714 Gartner Says Sales of Mobile Devices in Second Quarter of 2011 Grew 16.5 Percent Year-on-Year; Smartphone Sales Grew 74 Percent, Accessed on: 2011-09-13 